\title{
Growth and electrical properties of organic GOA crystals
}

\author{
Dr.(Mrs)Jyotsna R Pandey \\ (Material Research Laboratory, Department Of Physics \\ Birla College, Kalyan- 421301 (University Of Mumbai.),India.
}

\begin{abstract}
A new organic NLO materials GOA was synthesized by slow evaporation method. Glycine and oxalic acid were combined in 3 different molar ratios to form glycine Oxalic acid (GOA) transparent crystals of sizes $(2-6 \mathrm{~cm})$ in 2 to 3 weeks time. The phase formation using XRD studies shows orthorhombic crystal structure. The electrical measurement on the crystals at various temperatures shows that the resistivity of all samples increases with increase in temperature initially and to remain stable to decrease finally. The dielectric constant and dielectric loss factor of crystals was studied as a function of frequency ranging from $100 \mathrm{~Hz}$ to $1 \mathrm{MHz}$ and recorded that dielectric constant and dielectric loss decreases with higher frequency value. In I-V characteristics as voltage raised the current increases almost linearly.
\end{abstract}

Keywords—dielectric constant, dielectric loss, electrical resistivity, Glycine, oxalic acid, ,

\section{INTRODUCTION}

In recent years organic electronics has emerged as one of the major field in material science. The intermolecular attraction in organic materials is found to affect the conducting, dielectric, magnetic and optical properties [1-3]. Further in the field of organic electronics the versatile functionality of organic materials have been essential to the unparallel performance increase in semiconductor storage and display at the consistently lower costs [4-6]. Nonlinear optical materials (NLO) possess properties of optical modulation, optical switching and optical memory. The organic crystals exhibit flexibility of molecular design needed for application with using suitable donor acceptor organic crystals having special properties of large optical non linearity, low cutoff wavelength in UV region. Organic materials are often formed by weak Vanderwaal's and Hydrogen Bonds and hence possess a high degree of delocalization [7-8]. Amino acids and their complexes belongs to a family of organic materials that find applications in nonlinear optics [9-10]. Many Amino acids individually exhibit nonlinear optical properties because they have donor $\mathrm{NH}_{2}$ and acceptor $\mathrm{COOH}$ with possibility of charge transfer . Some Amino acids used as dopant enhance material properties like NLO and ferroelectric properties [11]. The second order optical nonlinearity, short transparency cutoff wavelength and stable physiochemical performance are needed in realization of most of the recent electronic applications. Amino acids find applications in devices like parametric amplifiers.

Glycine Oxalic Acid (GOA) crystal has been successfully grown by slow evaporation of solution containing the stoichiometric ratios of its components. The amino acid $\alpha$ - glycine $\left(\mathrm{NH}_{2} \mathrm{CH}_{2} \mathrm{COOH}\right)$ is the only protein forming Amino acid without centre of chirality's [12]. Grown crystals were characterized by various techniques. In the present study the resistivity of mixed glycine crystals for 3 different ratios was measured. The dielectric constant and dielectric loss factor were measured at frequencies ranging from $100 \mathrm{~Hz}$ to $1 \mathrm{MHz}$. This paper presents a comparative analysis of the samples with respect to its structure and electrical behavior.

\section{II.}

\section{EXPERIMENTS}

\subsection{Growth of GOA (3:1, 2:1, 1:1) crystals:}

GOA (Glycine oxalic acid) crystals were grown by slow evaporation technique. Glycine and Oxalic acid (monohydrate) both of AR grade supplied by Merck, India were taken in the respective molar ratios. Saturated solution was prepared and filtered using WHATMAN $110 \mu \mathrm{m}$ filter paper and subjected to slow evaporation at room temperature. Transparent and good crystals of appreciable sizes were obtained in 2 to 3 weeks times.(Figure1).The $\mathrm{P}_{\mathrm{H}}$ values and sizes of crystals noted for all these 3 different combinations are tabulated in Table 1. 

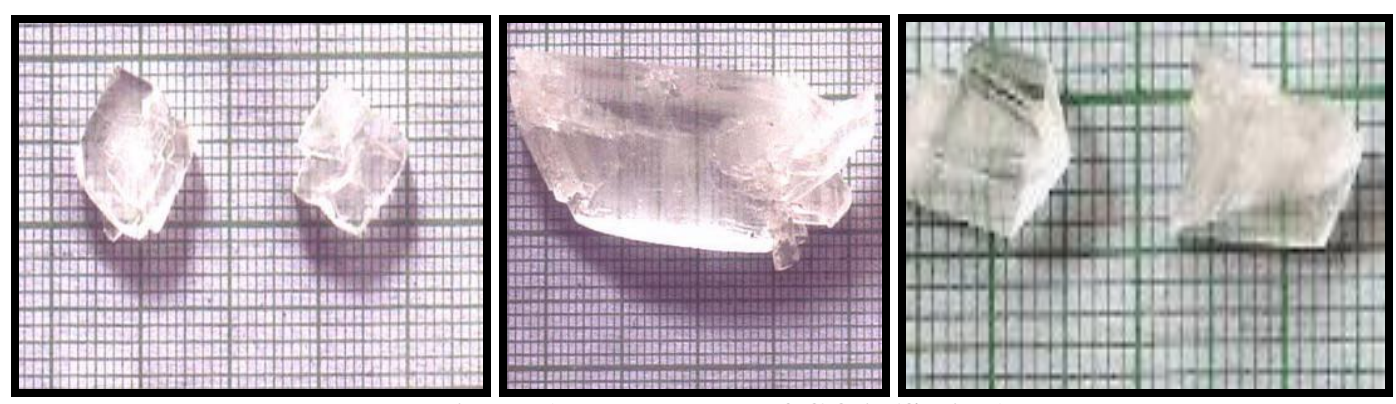

Figure 1: Photograph of GOA (Series1)

Table1: Parameters of GOA (series1)

\begin{tabular}{llll}
\hline Sample & PH & Sizes $(\mathrm{cm})$ & $\begin{array}{c}\text { Shape } \\
\text { Elongated }\end{array}$ \\
GOA3: 1 & 4.6 & $2-3 \mathrm{~cm}$ & Elongated \\
GOA2: 1 & 5.0 & $4-6 \mathrm{~cm}$ & Elongated \\
GOA1: 1 & 4.7 & $1-3 \mathrm{~cm}$ & \\
\hline
\end{tabular}

After the growth of the crystals of GOA $(3: 1),(2: 1),(1: 1)$, they are subjected to various physical and electrical characterizations.

2.2 To measure electrical properties of grown GOA (series) crystals electronic grade silver paste was applied on either surface of sample which acted as electrode. The experimental setup for performing dielectric studies has been discussed [12-13]. The capacitance and dielectric loss were measured for different frequencies from $100 \mathrm{~Hz}$ to $1 \mathrm{MHz}$ with sophisticated HP LCZ 4277 A METER.

\section{RESULTS AND DISCUSSION}

\subsection{Powder X-ray diffraction analysis_-}

Powder X-ray diffraction analysis was carried out on Panalytical X-pert MPO PRO from $20^{\circ}$ to $80^{\circ}$ with characteristics $\mathrm{Cu}-\mathrm{K} \alpha$ radiation with $\lambda=1.5418 \mathrm{~A}^{0}$. An interacative powder diffraction data interpretation and indexing program version 2.2 by E.Wu,school of physical sciences, Flinders university of south austrilia Bedford park,S.A.5042 Software program was used to assign planes and determine the lattice parameters for all the grown GOA (series1) crystals. The GOA crystals belong to the orthorhombic symmetry with lattice parameters as shown in table 2 .

The variation of lattice parameters with varying concentration of oxalic acid are depicted in figure 2. The ' $a$ ' and ' $c$ ' parameters shows increase as the amount of oxalic acid is raised where as the ' $b$ ' parameter passes through a minimum for the (GOA2) sample. The parameter ' $a$ ' and 'c' are seen to vary linearly while the parameter 'c' shows nonlinear variation with concentration of oxalic acid.

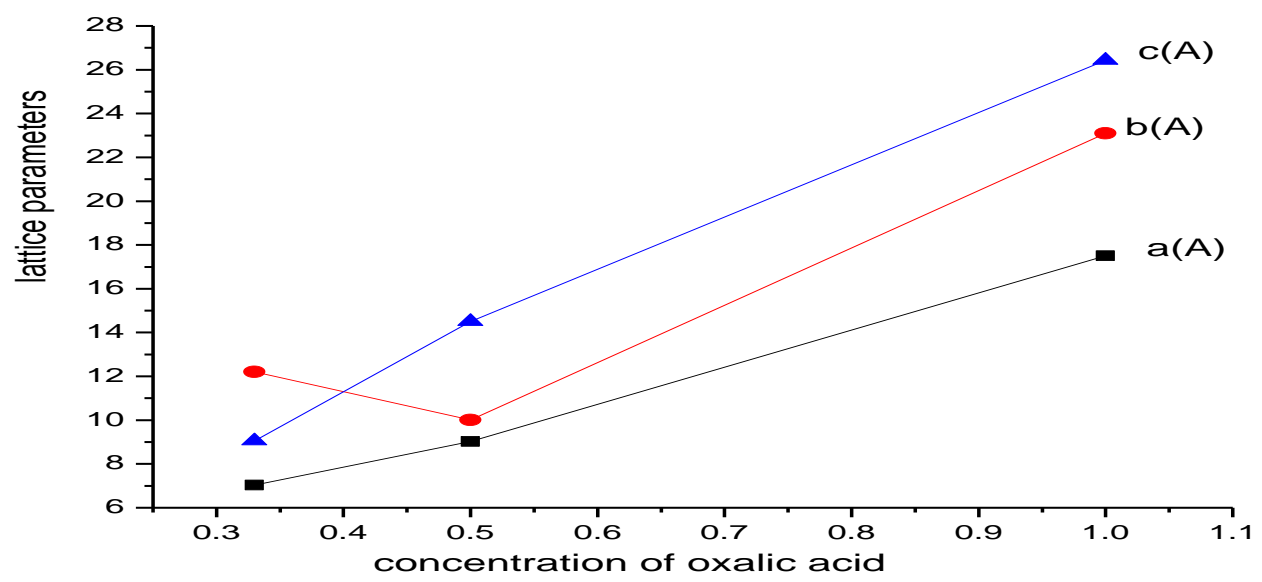

Figure. 2 Effete of concentration of oxalic acid on lattice parameters 
Table 2 : X-Ray Diffraction - Lattice parameter values

\begin{tabular}{llllll}
\hline Sample & $\mathrm{a}\left(\mathrm{A}^{0}\right)$ & $\mathrm{b}\left(\mathrm{A}^{0}\right)$ & $\mathrm{c}\left(\mathrm{A}^{0}\right)$ & Volume & Structure \\
GOA3: 1 & 7.035 & 12.20 & 9.05 & 777.33 & Orthorhombic \\
GOA2: 1 & 9.02 & 10.01 & 14.50 & 1309.21 & Orthorhombic \\
GOA1: 1 & 17.50 & 23.10 & 26.43 & 10707.45 & Orthorhombic \\
\hline
\end{tabular}

\subsection{Current-voltage characteristics-}

The I-V characteristics of grown series of GOA (series1) crystals have been determined. The I-V characteristics of GOA (series1) shown in figure 3. It is seen that as the voltage is increased the current increases almost linearly and the maximum and minimum value of current depends on the concentration of oxalic acid.

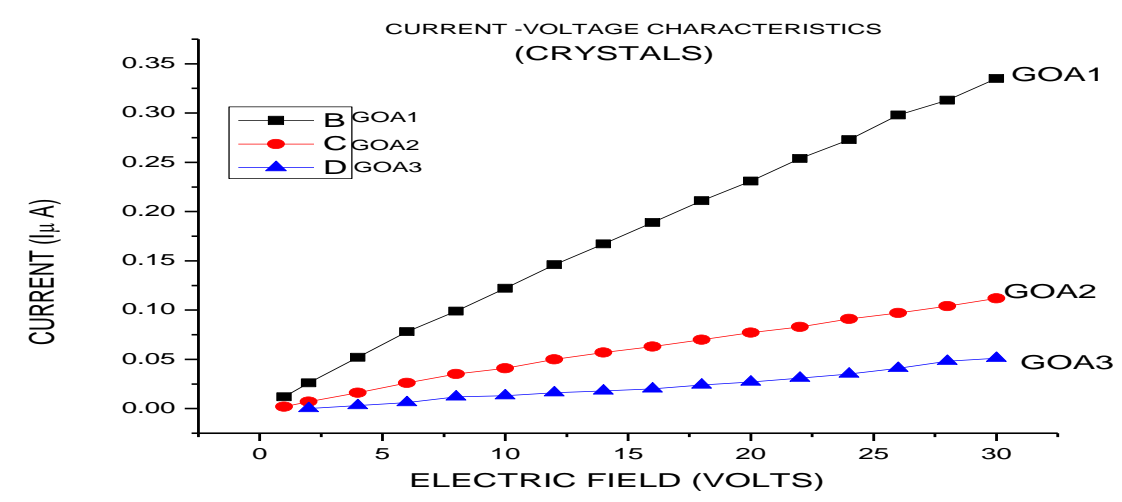

Figure 3. I-V Characteristics for GOA (series1)

\subsection{Resistivity with temperature}

The resisitivity is that region which is very sensitive to the impurity and lattice defects which was introduced in the process of crystal growth. The electrical resistivity was measured over the temperature $330 \mathrm{~K}$ to $440 \mathrm{~K}$. The appearance of resistivity peaks seems to be connected with crystal thickness and the solvent used. The conductivity is reciprocal of resistivity. The resistivity curves as shown in figure 4 . The resistivity are of order of $10^{7} \Omega \mathrm{cm}$ for GOA sample and their least-value have been observed for GOA2 sample followed by GOA3 and max value for GOA1.

The resistivity of GOA (series1) tabulated in table no 3. The lower value of resistivity for GOA2 suggesting conductivity by hydrogen atom and which depends upon the concentration of oxalic acid. For GOA (series) resistivity is seen to fall at temperatures $382{ }^{\circ} \mathrm{C}, 375{ }^{\circ} \mathrm{C}$ and $400{ }^{\circ} \mathrm{C}$. Less number of charge carriers are available for conduction with decrease in concentration of oxalic acid At theses temperatures the thermal charge carriers are set to free to become constant at temperature nearly $410{ }^{\circ} \mathrm{C}$.

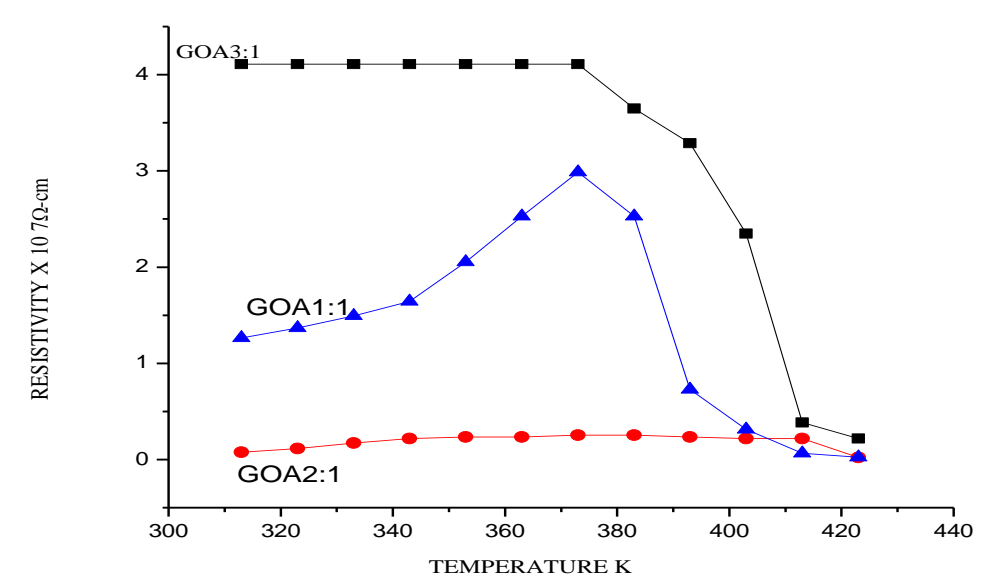

Figure 4 :Resistivity - Temperature Curves for GOA(series 1) 
Table 3 : Values of Resistivity for GOA ( series1)

\begin{tabular}{cc} 
& Table 3 : Values of Resistivity for GOA ( series1) \\
\hline Sample & Resistivity $\times 10^{7} \Omega-\mathrm{cm}$ \\
GOA3: 1 & $4.1094 \times 10^{7} \Omega-\mathrm{cm}$ \\
GOA2: 1 & $0.0764 \times 10^{7} \Omega-\mathrm{cm}$ \\
GOA1: 1 & $1.1730 \times 10^{7} \Omega-\mathrm{cm}$ \\
\hline
\end{tabular}

\subsection{Dielectric constant with frequency}

The dielectric constant value is a quantity which depends upon susceptibility of material in the applied electric field. The dielectric constant of insulator originates from both electronic polarization and molecular polarization. The dielectric constant is normally less than 3 above $10^{8} \mathrm{~Hz}$ with only electronic polarization present and molecular orientation may be becomes a contributing factor below $\approx 10^{8} \mathrm{~Hz}$. [14-15-16-17]. Figure 8 shows variation of dielectric constant with frequency for GOA (series). The dielectric constant has higher value in lower frequency region and it decrease with applied frequency up to $1 \mathrm{MHz}$. The maximum dielectric constant is '2300' for GOA3 and minimum dielectric constant '580' for GOA1. The higher value of dielectric constant at lower frequencies may be due to presence of all four polarization namely space charge, orientation electronic and ionic polarization and its low value at higher frequency maybe due to space charge polarization. [18]. As material undergo any of polarization can bind a certain amount of charge on the surface of material .The higher the polarization, the higher the bound charges on the surface and the higher the dielectric constant $(\mathrm{K})$ of material Figure 6 shows variation of dielectric constant with frequency for GOA (series1).

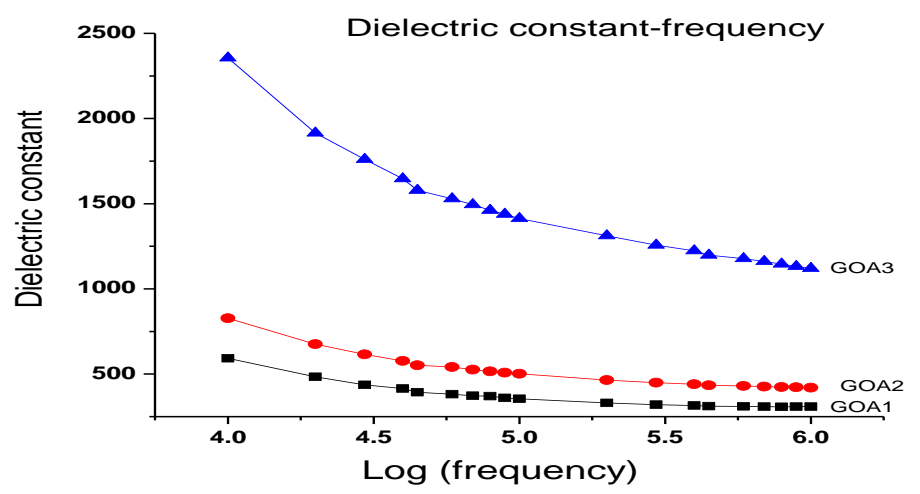

Figure 5 : Dielectric constant - $\log$ (frequency) for GOA ( series 1)

\subsection{Dielectric loss with frequency}

The dielectric loss depends upon the frequency. If the electrical field oscillates, the charge move back and forth but these displacement are extremely small ( $<1 \mathrm{~A} 0)$. If they occur for longer time they cause dielectric loss. [19-20]. Dielectric loss is not good to store charges in capacitor. Figure 6 shows value of dielectric loss with frequency for GOA (series1).The low value of dielectric loss at higher frequency for these crystals suggests that the samples posses' enhanced optical quality with lesser defects and these parameters plays a vital role for nonlinear optical material in their applications. The loss was found initially to decreased with frequency and later remained almost constant with the range of frequencies up to $1 \mathrm{MHz}$.

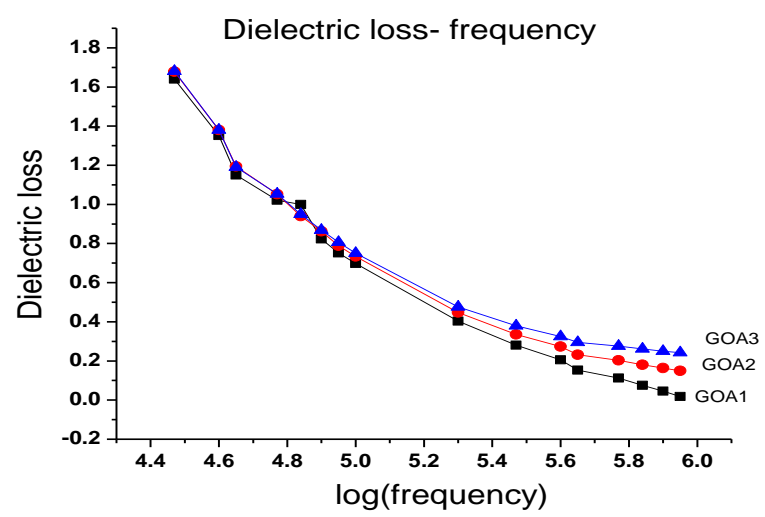

Figure 6: Dielectric loss - log (frequency) curves for GOA (series1) 


\section{CONCLUSIONS}

1. New organic GOA (glycine oxalic acid) crystals of appreciable sizes were grown from solution method in 2- 3 weeks time.

2. XRD studies reveal orthorhombic crystal structure. The lattice parameters ' $a$ ' and 'c' vary linearly with increase in concentration of oxalic acid.

3. The resistivity are of order of $10{ }^{7} \Omega$-cm and resistivity is seen to fall at temperatures $382{ }^{\circ} \mathrm{C}, 375{ }^{\circ} \mathrm{C}$ and $400{ }^{\circ} \mathrm{C}$.

4 The dielectric constant with temperature does not exhibit any specific phase transitions /anamoly.

5. The variation of dielectric constant and loss factor shows decrease in value as Frequency increase.

6. Variation of dielectric constant and loss factor is attributable to space charge polarization and orientation of domains.

\section{REFERENCES}

[1] G.Malliaras and R. H. Friend; Science Today 58(2005) 53

[2] D. R. Gamota, P. Brazis, Printed Organic Electronics, (Kluwer Academic,), 2004

[3] H. Okamota, T.Mitani, Phys. Rev B 43 (1991)

[4] Sachio Horiuchi, Tatsuo Hasegawa, Journal of the Physical Society of Japan, 75, 5 (2006)

[5] M.Beatrice,Crystal.Res.Tech., 7 (2006), 712-717

[6] N. Nakatani , Jpn. .J.Appl.Phys. 29 (2038) (1990)

[7] N.P.Rajesh, C.Mahadevan, MaterialLetters 55, (2002), 394-396

[8] D. K Burgate ,S.H Deshmukh, Indian Journal of pure and Applied Physics, 42, (2004), 533-538

[9] K. Meera A.K. Tripathy, Journal of Crystal Growth Vol 260, (2004), 414-421

[10] S .Dhanuskodi and J. Ramajothi, Crystal Res . Technol 39, 7, (2004), 592-597

[11] S.Aravozhi, R.Jayavel. Material Research Bulletin 32, 11, (1997),503-1513

[12] G.Sajeevkumar, B.S.Ramadevi, Bull Mater.Sci, l27,4, (2004), 323-325

[13] S.J.Joshi , M.J.Joshi, Bull.Mater.Sci 29, 3 , (2006), 307-312

[14] S.A.Martin Britto Dhas \& S.Natrajan, Optics Communications 12 (2007), 1-5.

[15] M.M.Khandpekar, Indian Journal of Pure and Applied Physics 41 (2003), 704-706.

[16] R.AnandaKumari \& R.Chandramani, Indian Journal of Physics 79 (2005), 31-35.

[17] A. Saxena, V. Gupta \& K.Sreenivas, Journal of Crystal Growth 263 (2004), 92-202.

[18] K.Ambujam, S.Selvakumar, D.PremAnand, G.Mohamed \& P.Sagayaraj Crystal.Res.Technol 41 (2006), 71-677.

[19] S.T.Vasan, F.M.Sannaninganavar, N.H.Ayachit \& D.K.Deshpande, Journalof Molecular Liquids 135 (2007),38-41.

[20] S.Ishwar Bhat, P.Mohan Rao, A.P.Ganesh Bhat \& D.K.Avasthi, Surface and Coatings Technology 158 (2002), 725728. 